\title{
ОБОБЩЕННОЕ ПРЕДСТАВЛЕНИЕ СОСТОЯНИЯ ФИЗИЧЕСКОЙ СИТУАЦИИ И ЕГО ПРИМЕНЕНИЕ В ЗАДАЧАХ ГИДРОДИНАМИКИ
}

\author{
(Представлена Н. Алумяэ)
}

\section{1. Основные понятия и положения}

Ансамбль и реализация физической ситуации. Пусть $a^{1}, \ldots, a^{N}-$ совокупность величин, фиксирующих на данном уровне описания состояние некоторой физической ситуации. Рассмотрим положение, когда значения $a^{1}, \ldots, a^{N}$ определяются неоднозначно. С подобной ситуацией приходится встречаться, например, при описании случайных процессов. Совокупность всевозможных состояний физической ситуации, соответствующих всевозможным наборам значений $a^{1}, \ldots, a^{N}$, будем называть ансамблем рассматриваемой физической ситуации, а ситуацию, соответствующую конкретному набору значений $a^{1}, \ldots, a^{N}$ - ее реализацией.

Обозначив принадлежность значений $a^{1}, \ldots, a^{N}$ к конкретной peaлизации нижним индексом $m, m=1, \ldots, M$, где $M$ - число возможных состояний (для начала остановимся на случае счетного числа возможных состояний), будем интерпретировать $a_{m}^{n}(n=1, \ldots, N)$ как компоненты векторов $\mathbf{a}^{n}$ в $M$-мерном евклидовом пространстве. Тогда ансамбль ситуации окажется представленным совокупностью $N$ векторов $\mathbf{a}^{1}, \ldots, \mathbf{a}^{N}$ в этом пространстве, а конкретная реализация ситуации - проекцией этих векторов на соответствующую ось системы координат.

Н аблюдаемая физическая ситуация. Фильтрация. Пусть, далее, е - вектор во введенном пространстве, все компоненты которого равны единице, а $\mathbf{A}$ - некоторый другой вектор, удовлетворяющий условию нормировки

$$
(\mathrm{Ae})=1 \text {. }
$$

Векторы в круглых скобках означают скалярное произведение $(\mathrm{ab})=$ $=\sum_{m=1}^{M} a_{m} b_{m}$. Сопоставим каждому вектору а при помощи вектора $\mathbf{A}$ число

$$
\langle a\rangle=(\mathbf{a A}),
$$

которое будем называть наблюдаемым значением величины (вектора) 
a. Аналогично, физическую ситуацию, соответствующую $\left\langle a^{1}\right\rangle, \ldots,\left\langle a^{N}\right\rangle$, будем называть наблюдаемой физической ситуацией. О величинах (векторах) с нулевым наблюдаемым значением будем говорить как о ненаблюдаемых.

Введем в рассмотрение вектор (аА)е. Очевидно, что (аA)e - вектор, наблюдаемое значение которого равно наблюдаемому значению вектора а, а вектор $\mathrm{a}^{\prime}$, определяемый как

$$
\mathbf{a}^{\prime}=\mathbf{a}-(\mathbf{a A}) \mathbf{e},
$$

- ненаблюдаемый.

Из соотношения (3) находим для а

$$
\mathrm{a}=(\mathrm{aA}) \mathrm{e}+\mathrm{a}^{\prime} \text {. }
$$

Первое слагаемое в правой части (4) будем называть наблюдаемой частью, а второе - ненаблюдаемой частью вектора а *. Учитывая, что разложение вектора а на наблюдаемую и ненаблюдаемую части зависит от конкретного выбора вектора А, будем называть вектор А вектором фильтрации, а операцию сопоставления вектору а величины $\langle a\rangle-$ операцией фильтрации.

К о р реля ц и я. Для введенного пространства справедливы, очевидно, все правила линейного векторного пространства (со скалярным произведением) $\left[{ }^{1}\right]$, в том числе и правила

$$
\begin{aligned}
& (\mathbf{A} \alpha \mathbf{a})=\alpha(\mathbf{A a}), \\
& (\mathbf{A}(\mathbf{a}+\mathbf{b}))=(\mathbf{A a})+(\mathbf{A b}) .
\end{aligned}
$$

Расширим введенное линейное векторное пространство до уровня линейной алгебры [ $\left.{ }^{1}\right]$ путем определения в нем операции умножения векторов

$$
\mathbf{a} * \mathbf{b} \equiv\left(a_{1} b_{1}, \ldots, a_{M} b_{M}\right)
$$

Рассмотрим операцию $(\mathbf{A a} * \mathbf{b})$. Используя для a и b разложение (4), с учетом (1), (5) и (6) находим

$$
(\mathbf{A a} * b)=(A a)(A b)+\left(\mathbf{A a}^{\prime} * \mathbf{b}^{\prime}\right) .
$$

Будем говорить, что величины а и $\mathbf{b}$ коррелируют, если $\left(\mathbf{A a}^{\prime} * \mathbf{b}^{\prime}\right) \neq 0$, и не коррелируют, если $\left(\mathbf{A a}^{\prime} * \mathbf{b}^{\prime}\right)=0$.

Правила вывода уравнений для наблюдаемой фи зи че ской с и ту а и и. В случае, когда а является функцией от некоторого параметра $\sigma$ (напр., от времени), и А не зависит явно от $\sigma$, нетрудно убедиться в выполнимости следующих правил:

$$
\begin{aligned}
& \left(\mathbf{A} \frac{\partial}{\partial \sigma} \mathbf{a}\right)=\frac{\partial}{\partial \sigma}(\mathbf{A} \mathbf{a}), \\
& \left(\mathbf{A} \int \mathbf{a} \mathrm{d} \sigma\right)=\int(\mathbf{A} \mathbf{a}) d \sigma .
\end{aligned}
$$

Соотношения (8), (9) вместе с соотношениями (5) и (7) представляют собой основные правила, по которым совершается переход от уравнений, описывающих отдельные реализации физической ситуации, к уравнениям, описывающим наблюдаемую физическую ситуацию.

\footnotetext{
* Вектор $\mathbf{a}^{\prime}$ можно определить и по-другому, например как $\mathbf{a}^{\prime}=\mathbf{a}-(\mathrm{aA}) \mathrm{A} / \mathrm{A}^{2}$, но выражение $\mathrm{a}^{\prime}$ в виде (3) приводит к некоторому упрощению итоговых формул.
} 
Случай непрерывного изменения возможных сос то ян и й. До сих пор мы рассматривали случай, когда переход от одного состояния к другому осуществлялся при дискретном изменении значений параметров состояния $a^{1}, \ldots, a^{N}$. Пусть, теперь, переход от одного состояния к другому происходит при непрерывном изменении $a^{1}, \ldots, a^{N}$. Параметризируем значения $a^{1}, \ldots, a^{N}$ совокупностью параметров $s_{1}, s_{2}, \ldots$. Тогда все векторы а переходят в функции $a\left(s_{1}, s_{2}, \ldots\right)$, вектор е - в единицу и формула скалярного произведения - в интеграл $\int_{\Sigma} a\left(s_{1}, s_{2}, \ldots\right) b\left(s_{1}, s_{2}, \ldots\right) \mathrm{d} s_{1} \mathrm{~d} s_{2} \ldots$, где $\Sigma$ - вся область изменения $s_{1}, s_{2}, \ldots$. Если для $a^{n}\left(s_{1}, s_{2}, \ldots\right)$ сохранить обозначение $\mathrm{a}^{n}$, то формулы (1)-(4), (5), (7) - (9) останутся справедливыми и в этом случае.

\section{2. Некоторые частные случаи операции фильтрации}

Для определенности ограничимся случаем непрерывного изменения возможных состояний.

О с р ед н ени е. Пусть $a=a(s), A=A(s)$, причем $A(s) \geqslant 0$ при любом $s$ из интервала определения $\left[s_{1}, s_{2}\right]$, и пусть $A(s) \mathrm{d} s$ есть вероятность того, что значения $s$ лежат в подынтервале $[s, s+\mathrm{d} s]$. Тогда операция фильтрации сводится к простой операции осреднения

$$
\langle a\rangle=\int_{s_{1}}^{s_{2}} a(s) A(s) \mathrm{d} s .
$$

Если $a(s)=s$, то (10) определяет статистическую среднюю величины $s$. Если $s$ - момент времени в интервале $\left[s_{1}, s_{2}\right]$, причем все моменты в этом интервале равноправны с точки зрения их выбора, то $A(s)=$ $=\left(s_{2}-s_{1}\right)^{-1}=$ const и (10) сводится к формуле осреднения по времени. Аналогично можно показать, что определение операции фильтрации включает в себя и осреднение по пространству.

Кл ассический пример операции фильтрации. Пусть мы имеем дело не с одним, а с целой совокупностью интервалов $\left[s_{1}, s_{2}\right]$ и пусть, кроме того, существует взаимно однозначное соответствие между членами этого множества интервалов и значениями некоторого параметра $s_{0}$, приобретающего значения из интервала $\left[s_{01}, s_{02}\right]$. Например, $s_{1}=s_{0}-T / 2, s_{2}=s_{0}+T / 2$, где $T=s_{2}-s_{1}=$ const. Тогда, вводя новую переменную $\sigma=s-s_{0}\left(\sigma=s_{1}-s_{0}, \sigma_{2}=s_{2}-s_{0}\right)$, в соответствии с (10) получим

$$
\langle a\rangle=\int_{\sigma_{1}}^{\sigma_{2}} a\left(s_{0}+\sigma\right) A\left(s_{0}+\sigma\right) \mathrm{d} \sigma .
$$

Если $A\left(s_{0}+\sigma\right)$ инвариантна относительно выбора $s_{0}$, то $A\left(s_{0}+\sigma\right)=$ $=A(\sigma)$ и формула (11) сводится к хорошо известной формуле фильтрации $[2,3]$.

\section{3. Обобщенное описание движения несжимаемой жидкости}

У р а внен и я $\mathrm{P}$ е й нольд с а. Применим сказанное выше к описанию движения вязкой несжимаемой жидкости. Будем считать движение среды в каждой отдельно взятой реализации течения полностью описанным (при заданных начальных, граничных и некоторых других 
условиях, гарантирующих единственность решения) уравнениями Навье-Стокса

$$
\varrho \frac{\mathrm{D}}{\mathrm{D} t} v_{i} \equiv-p_{, i}+\mu v_{i, j j}+\mathrm{\varrho} f_{i}
$$

Здесь $\mathrm{g}$ - плотность среды; $v_{i}, f_{i}-$ компоненты полей скорости и плотности внешних массовых сил; $\mu$ - коэффициент молекулярной вязкости среды; индекс после запятой означает дифференцирование по соответствующей координате, по повторяющимся индексам производится суммирование.

Пусть, далее, $a^{1}, \ldots, a^{N}$ - полная совокупность параметров и (или) полей, задание которых эквивалентно заданию всех требуемых условий так, что каждой конкретной совокупности значений $a^{1}, \ldots, a^{N}$ соответствует единственная реализация течения.

Перейдем от описания конкретной реализации течения к описанию наблюдаемых значений ансамбля реализаций течения. Для этого перейдем от $a^{1}, \ldots, a^{N}$ к векторам состояния $\mathrm{a}^{1}, \ldots, \mathrm{a}^{N}$ и от $\varrho \frac{\mathrm{D}}{\mathrm{D} t} v_{i}$, $-p_{, i}, \mu v_{i, j j}, \varrho f_{i}$ к соответствующим векторам состояния $\mathbf{b}_{i}, \mathbf{c}_{i}, \mathbf{d}_{i}, \mathbf{e}_{i}$. Очевидно, что при этом, согласно (12),

$$
\mathbf{b}_{i}=\mathbf{c}_{i}+\mathbf{d}_{i}+\mathbf{e}_{i} .
$$

Тогда с учетом смысла $\mathbf{b}_{i}, \mathbf{c}_{i}, \mathbf{d}_{i}, \mathbf{e}_{i}$ и правил (5), (7) и (8) имеем

$$
\left.\mathrm{Q} \frac{\mathrm{D}}{\mathrm{D} t}\left\langle v_{i}\right\rangle=-\langle p\rangle_{, i}+\mu\left\langle v_{i}\right\rangle_{, j j}-\mathrm{\varrho}\left\langle v_{i}^{\prime}{ }_{i}{ }_{j}{ }_{j}\right\rangle+\mathrm{Q}+\mathrm{f}_{i}\right\rangle \text {. }
$$

Уравнения типа (14) хорошо известны в гидродинамике как уравнения Рейнольдса, названные так в честь О. Рейнольдса, который вывел эти уравнения для описания осредненного турбулентного течения. Инвариантность уравнений (14) относительно конкретного выбора А означает в принципе обобщение этих уравнений. Из сказанного отнюдь не следует, что обобщенное в принятом смысле описание течения вообще не зависит от А. Выбор А весьма существен как при задании начальных и граничных условий уравнениям (14), так и при формулировке конкретной связи $\left\langle v_{i}^{\prime} v_{j}^{\prime}\right\rangle$ с кинематическими полями течения, необходимой для замыкания уравнений (14).

Авторы признательны Ю. В. Немировскому за обсуждение работы и ценные замечания.

\section{Л ИТ Е Р А Т Р А}

1. Корн Г., Корн Т., Справочник по математике, М., «Наука», 1970.

2. О з м и дов Р. В., Горизонтальная турбулентность и турбулентный обмен в океане, М., «Наука», 1968.

3. Я м по льски й А. Д., Океанология, V, вып. 5, 769-778 (1965).

Институт термофизики и электрофизики Академии наук Эстонской ССР
Поступила в редакцию 21/IX 1979 


\section{A. TOOMPUU, J. HEINLOO}

\section{FOUSIKALISE SITUATSIOONI OLDISTATUD ESITUS JA SELLE RAKENDAMINE HUDRODUNAAMIKA OLESANNETES}

On esitatud üheselt määramata parameetritega füüsikalise situatsiooni kirjeldamise meetod. Selle rakendamisel hüdrodünaamilise situatsiooni korral on tulemuseks Reynoldsi vôrrandid, kuid erinevalt nende tavalisest interpretatsioonist osutuvad tuletatud võrrandid suvalise filtreerimisoperatsiooni suhtes invariantseteks.

\section{A. TOOMPUU, J. HEINLOO}

\section{THE GENERALIZED REPRESENTATION OF A STATE OF A PHYSICAL SITUATION AND ITS APPLICATION TO PROBLEMS OF HYDRODYNAMICS}

An approach is developed for describing a physical situation in the case if its singlevalued fixation is impossible. (Such a situation takes place in the special case of describing stochastic processes).

A state vector is assigned to each one of the parameters the total amount of which uniquely fixes the given physical situation. Components of the state vector are equal to eventual values of the parameter (quantity). The operation of filtration is defined by (2) where A is the filtration vector, the sum of components of which is equal to 1 (1).

Derived rules (5), (7), (8), (9) enable to get equations for filtered quantities on the basis of equations describing a single realization of a physical situation. It is shown that the applying of this approach to Navier-Stokes equations (12) brings us to Reynolds equations (14). Unlike the traditional interpretation of these equations, the derived equations are invariant in respect to the interpretation of the origin of the set of the eventual values of hydrodynamical quantities and in respect to the filtration vector $\mathbf{A}$ in the proposed treatment. 